\title{
Desde el Tratado de Paz y Amistad de 1984 al Tratado de Maipú de 2009: Un Proceso eVolutivo INSTITUCIONALMENTE CONSOLIDADO*
}

\author{
Since the Treaty of Peace and Friendship of 1984 to the Treaty of Maipu \\ of 2009: an Institutionally Consolidated Evolutive Process
}

\section{DIEGO JIMÉNEZ CABRERA}

Universidad de Santiago de Chile

\begin{abstract}
RESUMEN
Argentina y Chile han desarrollado desde 1990 la etapa de cooperación más profunda de toda su historia republicana. Para hacerla posible, la élite política de cada país reformuló su visión del otro, reestructurando al efecto el espacio geopolítico referencial que cada una tenía en relación con la percepción del papel de su respectivo país en la sociedad internacional. Así, las instituciones binacionales autónomas argentinochilenas son la culminación de una transición hacia una nueva etapa en las relaciones bilaterales, marcadas por la construcción e implementación de un discurso geopolítico dirigido a formar un sistema institucional binacional autónomo.
\end{abstract}

Palabras clave: Espacio geopolítico, teoría de toma de decisiones, discurso geopolítico, Chile, Argentina.

\begin{abstract}
Since 1990 Argentina and Chile have been developing the deepest stage of cooperation in its republican history. In order for both countries to be able to make that kind of cooperation viable, Argentina's and Chile's political elites reformulated their views of each other, reconstructing the referential geopolitical space that each country had about its role in the international society. Thus, autonomous bi-national institutions are the outcome of a transition process towards a new stage in the case of Argentinian-Chilean relations, marked by the construction and implementation of a geopolitical discourse oriented to create such institutions.
\end{abstract}

Key words: Geopolitical space, decision-making theory, geopolitical discourse, Chile, Argentina.

* Agradezco los comentarios de los revisores anónimos de RCP, así como la invaluable ayuda prestada al suscrito por los siguientes investigadores: Dr. Alfonso Dingemans (USACH), Dr. Juan Eduardo Mendoza (UdeC), Mg. Karen Manzano (UdeC), Mg. Constanza Jorquera (USACH) y Mg. Esteban Arratia (ANEPE). Asimismo, el autor agradece el financiamiento de la Fundación Volcán Calbuco por medio de su programa de becas de magíster (2014-2015) para desarrollar el presente artículo. 


\section{INTRODUCCIÓN}

Después de casi 70 años de concordia, Argentina y Chile enfrentaron el conflicto del canal Beagle, en el que ambos países estuvieron a horas de la guerra en 1978. Empero, la mediación papal y la presión de EE.UU. instaron a las partes a emprender las negociaciones que dieron forma al Tratado de Paz y Amistad (1984). Este tratado contribuyó a descomprimir las presiones existentes en ambos países.

Aceptada la mediación, pasaron cinco años de alta tensión. Chile continuaba negociando una solución definitiva a la controversia apuntando a obtener el respeto total del Laudo Arbitral de 1977, mientras Argentina respondía escuetamente a los avances de las negociaciones, en razón de las divisiones internas que el tema causaba en las FF.AA. La ruptura de esta dinámica se produjo con la derrota argentina en la Guerra de las Malvinas (1982) y la asunción de Raúl Alfonsín como Presidente de Argentina (1983), quien dispuso los medios para firmar y ratificar el Tratado de Paz y Amistad tras la aplicación de un plebiscito no vinculante que contó con el $78 \%$ de apoyo para la propuesta papal y $22 \%$ en contra, sobre un universo del $74 \%$ del padrón electoral de la época.

Tras una distensión de seis años de la relación, esta adquirió, al alero del Tratado de Paz, un nivel de intensidad diferente una vez culminados dos procesos: 1) la llegada de Carlos Menem (1989-1999) a la presidencia argentina, y; 2) la asunción a la presidencia de Chile de Patricio Aylwin (1990-1994). La convergencia entre ambos gobiernos democráticos dio inicio a una de las etapas más fecundas en la historia binacional la que, para efectos de este estudio, culmina con el término del primer gobierno de Cristina Fernández (2011).

Para comprender los procesos del período 1989-2011, es importante apreciar la influencia de tres factores: 1) la evolución del discurso geopolítico de la élite política de Argentina y Chile, por medio de la revisión de la imagen del otro mediante las declaraciones presidenciales conjuntas y los tratados bilaterales; 2 ) el desarrollo de la agenda bilateral en las cuatro áreas clave de política de la relación (Seguridad y Defensa, Economía, Política e Integración Física), para ello se dispone de una descripción histórica de la relación en las áreas señaladas y; 3 ) la institucionalización de sus entidades binacionales, a partir de un análisis del Tratado de Maipú.

La hipótesis que defiende este artículo es que esta etapa de la relación argentinochilena es la primera en la que existe realmente un proceso de integración, por cuanto el discurso geopolítico de la élite política de ambos países se ha cristalizado en un sistema institucional binacional autónomo, cuyo núcleo es el Tratado de Maipú, dando lugar a una nueva etapa en el desarrollo de una relación que había comenzado con la transición institucionalizadora representada por el Tratado de Paz y Amistad.

\section{EL ESTADO DEL ARTE DE LA RELACIÓN ARGENTINO-CHILENA}

Si bien la producción científica argentino-chilena estuvo principalmente sustentada en la historia, la geografía y la geopolítica durante tres cuartas partes del siglo XX, y orientada a amalgamar una visión cimentada en la desconfianza mutua (Lacoste, 2003), 
en la década de los 80 y los 90 existieron cambios importantes. Mientras en Argentina surgen los trabajos de Norberto Ceresole (1987), Roberto Russell (1992) y Carlos Escudé (1992), en Chile fueron las obras de Heraldo Muñoz (1989) y Alberto van Klaveren (1988) las que comenzaron a mostrar enfoques orientados a construir principios de política exterior funcionales a la conservación de la democracia, la implementación de procesos de apertura económica y política con los vecinos, y a comprender las relaciones de Argentina y Chile con las mayores potencias de la sociedad internacional. Aquellos autores marcaron la pauta del desarrollo teórico de la política exterior de ambos países durante los 90 .

Durante los últimos 17 años (2000-2016) las investigaciones acerca de la relación argentinochilena cobraron mayor fuerza a partir de los resultados obtenidos por Argentina y Chile en su proceso de integración. Los trabajos de los argentinos Pablo Lacoste (2003) y Miryam Colacrai (2010: 305-327) muestran elementos relevantes relacionados a los rasgos, aspiraciones e intereses comunes de Argentina y Chile, tanto en lo económico como en lo político y cultural. A estos trabajos se suman los de los chilenos Eduardo Rodríguez (2006) y Luis Maira (2010), y la obra conjunta de Ricardo Cicerchia (Argentina) y Eduardo Cavieres (Chile) (2012). En efecto, la cooperación académica entre ambos países ha sido notable.

No obstante, el estudio del impacto del Tratado de Maipú en la relación argentinochilena ha sido menor, destacándose las obras de Alejandro Simonoff (2013) y María Elena Lorenzini (2013) en el caso argentino, y de Luis Maira (2010) en Chile. Asimismo, la mayoría de quienes han estudiado la relación argentino-chilena consideran que la nueva etapa en dicha relación la marca el Tratado de Paz y Amistad de 1984. Al respecto, este trabajo postula que este último tratado representa solo una transición en la que ambos países, con diferencias, contradicciones, errores y omisiones, trabajaron para formar un proceso inspirado por una imaginación y un discurso geopolíticos de complementación, que se caracterizó por un alto nivel de estabilidad desde el punto de vista de la configuración de instituciones binacionales formales. Para comprender este problema de investigación se empleará el enfoque de geopolítica crítica.

\section{TOMA DE DECISIONES: DEL ESPACIO Y EL DISCURSO GEOPOLÍTICOS}

La relación argentino-chilena alcanzó una alta tensión durante los años 60 y 70 . En la geopolítica de Guerra Fría, la concepción de espacio político (Gallois, 1992: 51-64) fue la que prevaleció, en cuanto a que el principal recurso de poder del Estado era el territorio, en un sistema internacional percibido bajo un esquema de bolas de billar. Así, la expansión es indispensable para la sobrevivencia de los Estados, tanto por tierra como por mar, aunque también lo es el adquirir una notoria influencia sobre otros Estados cuando la expansión es imposible (Lacoste, 2000: 65-92).

Esta visión del territorio se proyectó a partir de una tensión creciente en la relación argentino-chilena desde fines de los años 40, con el desarrollo de las negociaciones relativas al litigio de Palena (Valenzuela, 1999: 13-80). En este período, ambos países 
se orientaron a consolidar una imagen del otro en una lógica de víctima/victimario mediante la difusión de tesis de fronteras que no correspondían a lo indicado por los antecedentes históricos sobre la materia (Lacoste, 2003). Así, los gobiernos respectivos utilizaron como un recurso común la construcción de la identidad nacional y la exaltación del nacionalismo mediante la creación y proyección de un enemigo externo (Pinto, 2010: 145-160) encarnado en el vecino. Este proceso alcanzó su punto cúlmine en diciembre de 1978. Posteriormente, se registró una baja en las hostilidades hasta 1984.

En el período 1978-1984, desde la academia de ambos países y sus respectivas élites políticas comenzó un proceso de reestructuración de la forma de visualizar el mundo en lo general, y las relaciones con el otro en lo particular. Sugerimos entonces que se produjo, en términos del geógrafo político británico-estadounidense John Agnew, un proceso de reestructuración de la imaginación geopolítica de los actores. Esta imaginación es definida por Agnew como:

[...] un sistema de visualización del mundo con hondas raíces históricas [...]. Se trata de una imagen elaborada del mundo y no de una imagen sencilla y espontánea que nazca de la mera contemplación del mundo desde el "sentido común". La imaginación geopolítica moderna, al ser un sistema de teoría y práctica, no ha existido ni existe en el vacío (2005: 7).

Este proceso de reestructuración de la imaginación geopolítica estuvo marcado por las negociaciones entre diversos asesores y decision-makers que contribuyeron a transformarla en la práctica, para luego consolidar esta transformación desde la teoría. ${ }^{1}$ Asimismo, se desarrolla un cambio vital en el concepto geopolítico del territorio: las aspiraciones de Argentina y Chile ya no se realizarían expandiéndose a costa del otro, sino que estarán enmarcadas en un proceso cooperativo que considera al territorio desde la óptica de un acceso preferencial.

Lo anterior implicaba cambiar la forma en la que se estudia el espacio y la dinámica espacial de los Estados. Así, se desarrolla el concepto de espacio geopolítico que José Felipe Marini define como "el área geográfica en cuyo seno actúan recíprocamente los factores geográficos y políticos que conforman una situación geopolítica que se desea estudiar o resolver" (1985: 45). Desde este prisma el territorio, aún si no es ocupado, posee igualmente un valor fundamental para el desarrollo nacional de Estados extranjeros y se actuará sobre él dentro de un marco de no intromisión en los asuntos de otros. Respecto de Argentina, el geopolítico argentino Norberto Ceresole señaló:

La seguridad externa del futuro argentino, de su proceso de cambio político y de transformaciones estructurales, no se agota en el Atlántico. La seguridad externa tiene otras dos dimensiones fundamentales, una en el plano continental y otra en el orden extracontinental.

1 Se puede observar en las cuentas públicas del gobierno de Raúl Alfonsín (1983-1989) una necesidad permanente de establecer relaciones pacíficas con Chile desde 1983. En una perspectiva teórica, uno de los pocos académicos disidentes de la visión de Osiris Villegas, José Rey Balmaceda y otros autores que pregonaban una visión de Chile como enemigo fue Norberto Ceresole (1944-2003), quien examinaba procesos de cooperación con Chile desde la segunda mitad de los años 80 . 
En el plano continental, nuestro destino como nación a la par que se consolida en su posición atlántica, deberá buscar vías de solución a través de una re-orientación de su política hacia el Pacífico. Porque limitarse simplemente a aceptar cualquier tipo de "alianza atlántica", aún en el supuesto de lograr un equilibrio militar respecto de Brasil, significaría congelar una situación que en el largo plazo favorecería solo a esa nación.

En el orden internacional, la única opción seria para reafirmar nuestro espacio político consiste en realizar una profunda apertura que deslimite (sic) y supere los actuales márgenes de dependencia (1987: 119).

Podemos deducir de lo anterior que, en el caso argentino-chileno, las aspiraciones geopolíticas han comenzado a tener lugar en espacios geopolíticos, no en el territorio per se, es decir, no buscan estos Estados obtener soberanía a partir del otro, sino poder contar con un acceso privilegiado a áreas geográficas funcionales al cumplimiento de sus respectivos objetivos geoestratégicos (Marini, 1985: 85-118). La consolidación de este proceso demanda dos condiciones: 1) que se desarrolle mediante el discurso geopolítico de la élite política de ambos países; y 2) que dicho discurso se materialice en instituciones políticas autónomas mediante un proceso de transformación de la imaginación geopolítica en una práctica dominante y "natural" para los actores del sistema (Uc, 2008: 111-3). Sin embargo, para lograr una comprensión teórica más completa del problema se requiere examinar la teoría de la toma de decisiones.

Según Graham T. Allison, son los intereses personales más que los del Estado los que mueven a los funcionarios de gobierno a ejecutar las decisiones de los altos niveles jerárquicos. Esto supone, de acuerdo con el Modelo del Actor Racional, que las decisiones se implementan con información perfecta. Aunque se requiere hacer dos comentarios al respecto: 1) la mayor dificultad radica en interpretar adecuadamente la información disponible, ya en esta etapa se generan las mayores distorsiones en torno a la ejecución de la política exterior; y 2) los funcionarios no solo se mueven por intereses propios, sino que existen ideas, valores e imago mundi que definen intereses intransables de los primeros respecto de sus superiores jerárquicos (Rhodes, 1994: 39-41). Por ende, los actores no son completamente racionales.

De esta manera, dependerá de la percepción que los decision-makers tengan de los demás participantes de un proceso lo que más influirá en la decisión a adoptar. No obstante, estos últimos no son conscientes de por qué perciben a los actores de una forma determinada, así como de las interpretaciones erróneas que hagan a partir de la información disponible, y que luego decodifican mediante la percepción. Ergo, los decision-makers no siempre toman decisiones racionales debido a la interpretación que hacen estos de la información recabada y procesada (Russet y Starr, 1990: 58-9), lo que puede llevar a sobredimensionar positiva o negativamente el poder real de la contraparte durante el proceso decisorio, fenómeno conocido como misperception. En efecto, al existir una mayor acumulación de experiencia a partir de la interacción con el otro se genera una apreciación menos distorsionada de la información relativa a este y sus objetivos durante el proceso de negociación, lo que hace posible que la imagen del otro se modifique, y con ello pueda 
existir una evolución de la imaginación geopolítica. Esto último acaba por traducirse en un ajuste evolutivo del discurso geopolítico. ${ }^{2}$

Sin embargo, lo anterior nos permite dimensionar el cambio en las percepciones, no así el efecto de la formación de instituciones en las relaciones entre países. Para ello se requiere incorporar el concepto de veto player, que "es un actor individual o colectivo cuyo acuerdo [o por regla de mayoría en el caso de los actores colectivos] es requerido para un cambio de política pública" (Tsebelis, 1995: 301). Asimismo, Tsebelis establece dos tipos de veto players: los partidarios y los institucionales. Mientras que los primeros se definen endógenamente por la estructura del sistema de partidos y las coaliciones gobernantes, los segundos son establecidos por la Constitución (Tsebelis, 1995: 304-5). Así, la característica distintiva de un veto player consiste en su capacidad para bloquear una política determinada (Tsebelis, 1995: 305), lo que también es aplicable a áreas de política (policy issue areas) que tengan estrechos vínculos con la política interna (Kastner y Rector, 2003: 2).

Si la creación de instituciones es un proceso formal de materialización del poder, esto implica lógicamente que cada institución, al dividir el proceso de toma de decisiones, es un potencial veto player (Mansfield, Milner y Pevehouse, 2007: 405). De este modo, mientras más veto players existan en una institución, esta tenderá a ser más estable y resistente a los cambios (Tsebelis, 1995).

Por otra parte, un Estado requiere ser consciente de su realidad geográfica, la que condiciona la aplicación de sus políticas para el desarrollo y, por tanto, necesita ajustar un proceso de integración a sus características políticas, económicas y geográficas particulares (Gómez, 1977: 227). Si bien en la evolución de la política mundial en los años 80 y 90 se ha desagregado la autoridad del Estado en el ámbito local y subnacional (Russell, 2010: 88-94), la integración demanda igualmente la convergencia de los intereses estatales si consideramos que desde el año 2001 las relaciones argentino-chilenas han estado marcadas por un regreso del Estado como protagonista de estas últimas (Russell, 2010: 94-102).

Si pensamos en los Estados como agentes conductores y ejecutores de la política exterior con una fisonomía basada en redes institucionales, entonces solo cuando existen instituciones políticas multilaterales es que aquellos se comprometen a ajustar sus instituciones bajo una modalidad ganar-ganar con otros Estados (Palomares, 2006). Así, podemos inferir que un proceso de integración solo existirá, en este caso, cuando se han formado instituciones binacionales autónomas que efectivamente conformen un sistema institucional. 


\section{LA AGENDA HISTÓRICA (1989-1994)}

Desde la firma del Tratado de Paz y Amistad hubo cambios radicales en la relación argentino-chilena: de haber estado a minutos de la guerra, ambos países pasaron a ser aliados estratégicos sobre la base de los pilares implementados por aquel tratado. Las condiciones de la relación en los 90 fueron diferentes a las de los 80: en ambos Estados se instalaron gobiernos democráticos, lo que contribuyó decisivamente a su acercamiento. Al respecto, con la primera declaración presidencial conjunta de 1990 buscaron

Reiterar enfáticamente, frente al actual escenario internacional, la vocación pacifista de sus naciones, orientadas por principios fundamentales como son la sujeción al orden jurídico internacional, la solución pacífica de las controversias, el no uso de la fuerza o la amenaza de su empleo, la integridad territorial de los Estados, la autodeterminación de los pueblos y la no intervención en asuntos que el derecho internacional reserva a la competencia privativa de los Estados, la fiel observancia de los tratados internacionales, la promoción de la democracia representativa y el respeto irrestricto de los derechos humanos (Declaración Conjunta argentino-chilena sobre la Relación Bilateral, 1990: 1).

Se reafirmaron los principios del Tratado de Paz y Amistad, con la salvedad de que ambos países se comprometieron a consolidar sus democracias para generar un proceso de integración multidimensional, que admitiera la solución pacífica de controversias junto con un avance progresivo de la relación en todos los ámbitos del nivel nacional, creándose un ambiente de mayor concordia para solucionar los 24 litigios limítrofes pendientes a esa fecha (Declaración Presidencial Conjunta sobre Límites entre la República Argentina y la República de Chile, 1991), quedando inconclusos los de Laguna del Desierto y Campos de Hielo. Así, mientras 22 de ellos fueron analizados por la Comisión Mixta de Límites para que las partes negociaran directamente una solución, se preparó el arbitraje de Laguna del Desierto.

La relación en el ámbito político se profundizó, suscribiéndose entre ambos países numerosos compromisos. Estos se extienden a ámbitos diversos, como la protección de inversiones, los acuerdos de complementación económica (ACE) y la constitución de comités de frontera (CDF) (1990-1995). Empero, el diferendo por la Laguna del Desierto generaba grandes expectativas en ambos Estados.

Una vez emitida la sentencia del 21 de octubre de 1994 -la que concedió a Argentina casi toda su pretensión de máxima-, el gobierno chileno comenzó, ante las presiones de la oposición para rechazarla, a preparar el recurso de revisión e interpretación, según lo contemplado en el Anexo I del Tratado de Paz y Amistad. Así, Chile ejerció el recurso ante el Tribunal Arbitral el 31 de enero de 1995, tras la sentencia emitida por este. El primero tuvo como resultado final el rechazo por tres votos a dos, y el segundo fue rechazado por cuatro votos contra uno, al aducir Chile errores de hecho y de derecho en la dictación de la sentencia de 1994 y en los procedimientos para su formulación (Tribunal Arbitral Internacional, 1995: 15). 
En principio, lo anterior no obstruyó el desarrollo de la relación bilateral, sino que dio cabida a un prolífico desarrollo en paralelo de los demás temas de la agenda bilateral, a partir del encapsulamiento del litigio de Laguna del Desierto a una solución estrictamente jurídica (Jiménez, 2012). Al efecto se suscribieron, entre otros, el acuerdo del Comité Parlamentario Conjunto (1991) y el Sistema de Información y Consulta Chileno-Argentino (Acuerdo por canje de notas argentino-chileno estableciendo un mecanismo de consulta, 1994). ${ }^{3}$ Asimismo, se sumó la Declaración Presidencial del 28 de agosto de 1992, que manifestó la voluntad política de ambos países de mejorar la relación bilateral. También se acordó el Plan Maestro General de Pasos Fronterizos (1992), dentro de la cual se consolidó una agenda de integración física que ya había comenzado con la constitución de los CDF Cristo Redentor (1987), Integración Austral (1991), NOA-Norte Grande (1991), Región de Los Lagos (1991), Agua Negra (1992), ATACALAR (1992) y Pehuenche ${ }^{4}$ (1993). En los pasos fronterizos inscritos en los CDF antes señalados, también se contempló la construcción de gasoductos, proyectos de transporte internacional, mineros y energéticos conjuntos que dan cuenta de la profunda vinculación entre las agendas de integración física con la agenda económica desarrollada al alero del ACE 16, firmado en 1991 (BIDINTAL, 2002: 11-52 y 135-54).

El ACE 16 rige desde 1992 a la fecha diversos ámbitos de la relación bilateral en lo económico y comercial a partir de un Comité de Complementación Económica guiado por el Comité Binacional Argentino-Chileno, y con su misma estructura orgánica. Esta institución es relevante en el desarrollo de la agenda bilateral, por cuanto se constituyó un sistema completo de relaciones económicas e integración física amparado en este instrumento y sus 28 protocolos complementarios. De estos últimos, los más gravitantes tuvieron lugar entre 1991 y 1995. Sus resultados fueron comentados y debatidos por comités políticos y académicos binacionales (BID-INTAL, 2002).

En el ámbito de Seguridad y Defensa se desarrolló una nueva agenda conjunta. Esta constó de numerosas reuniones entre los principales representantes de todas las ramas de las FF.AA. de ambos países. Así, tuvieron lugar 20 eventos conjuntos de defensa (Runza, 2004: 10) que fueron claves en la construcción de nuevas confianzas entre ambos países (ferias internacionales aeronáuticas, reuniones de Estados Mayores, etc.), especialmente desde que se implementaran las Medidas de Confianza Mutua (MCM) entre Argentina y Chile (1992), que apuntaban a desmantelar los elementos constitutivos más prevalentes de las percepciones de amenaza de ambos países respecto del vecino (Mendoza, 2004: 110-21), lo que permitió un avance de la agenda bilateral en los cuatro ámbitos comentados.

3 El Sistema de Información y Consulta consiste en la institucionalización de un conjunto de reuniones y encuentros entre los cancilleres, vicecancilleres y otras autoridades políticas. Este acuerdo es relevante en razón de que este permitió actualizar acuerdos y generar instrumentos jurídicos con la misma validez que un tratado en materias propias del Poder Ejecutivo de ambos países vía intercambio de notas entre los respectivos cancilleres.

4 Fue subcomité hasta el año 2002, en que adquirió el estatus de CDF. 


\section{CHILE EN EL ATLÁNTICO Y ARGENTINA EN EL PACÍFICO5 (1995-2002)}

La controversia de Campos de Hielo Sur se insertó en la agenda bilateral con la Declaración Presidencial de agosto de 1991, en que se señaló el fin de construir una frontera de paz a partir de la solución de 24 problemas limítrofes. Así, ambos países acordaron una traza de 17 vértices conocida como "la poligonal" 6 (Acuerdo entre el Gobierno de la República Argentina y el Gobierno de la República de Chile para precisar el límite en la zona comprendida entre el monte Fitz Roy y el cerro Daudet, 1991). Si bien esta ingresó el 2 de agosto de 1991 a trámite legislativo en ambos países, se vio truncada rápidamente, ya que ese año empezó el litigio de Laguna del Desierto.

Si bien fueron aplicados los compromisos suscritos en el período 1990-1994, se generó el siguiente problema: el Congreso Nacional argentino estaba en contra de la poligonal, y su homólogo chileno manifestó la misma disposición. Al respecto, aunque el gobierno de Menem hizo todas las gestiones posibles para proseguir con el trazado antes señalado, la poligonal no logró salvarse de seis años de letargo (Eissa, 2010: 35-54). En cuanto a Chile, el Congreso en conjunto rechazó rápidamente la iniciativa, a la espera del proceso de Laguna del Desierto.

Sin embargo, la sentencia de 1994 cambió el panorama de la relación: apareció un nuevo punto de inflexión a partir de las protestas de la oposición en ambos países. En Chile, el descontento ante el fallo fue manifestado por la prensa, tras la emisión de un comunicado por el que el gobierno demuestra públicamente su disposición a acatarlo, y en Argentina se difundió profusamente la victoria de Laguna del Desierto. Estas situaciones dieron origen al cuestionamiento argentino hacia Chile por la validez del recurso de revisión interpuesto, emitido por la prensa el 15 de marzo de 1996 (Illanes, 2003: 203-6). Así, mientras que el gobierno chileno no estaba dispuesto a someter la controversia de Campos de Hielo Sur a un arbitraje en razón del resultado de la sentencia de 1995 y a los cuestionamientos de la oposición, el gobierno argentino temía que, de darse un arbitraje, Chile pudiera salir favorecido por tener derechos mejor fundados sobre la región (Eissa, 2010: 35-54).

De aquel proceso resultan relevantes las mayorías logradas en ambas Cámaras del Congreso chileno por la Concertación, lo que facilitó las negociaciones. Esto permitió conducir una política exterior más fluida, con mecanismos claros de presión y diálogo para lograr una solución vía negociación directa. Al respecto, el gobierno chileno dio señales para instar al gobierno argentino a intensificar las gestiones ante el Congreso, como firmar el ACE 35 (1996), el Acta de Constitución del Grupo de Trabajo sobre Medidas de Integración para la Zona Austral (1996), el Tratado de Integración y Complementación Minera (1997), y el apoyo a la demanda argentina sobre las islas

5 Consigna expresada por Carlos Saúl Menem el 11 de diciembre de 1996 tras la firma, junto a Eduardo Frei Ruiz-Tagle, del Protocolo Adicional al Tratado sobre los Hielos Continentales entre Argentina y Chile en el Patio de los Cañones del Palacio de La Moneda (Santiago).

6 Consiste en la propuesta de delimitación de Campos de Hielo Sur de junio y julio de 1991, cuya extensión abarcó desde el monte Fitz Roy hasta el cerro Daudet, y que buscaba balancear las aspiraciones de Argentina y Chile mediante una distribución territorial equitativa (Eissa, 2010: 13-23; Manzano, 2015: 100-7). 
Malvinas, convirtiéndose este en el acto más relevante dentro de aquellos destinados a movilizar las negociaciones en el gobierno argentino (Declaración Presidencial Conjunta entre la República Argentina y la República de Chile, 1999: 3). También fue clave la conducción de las negociaciones nacionales e internacionales por parte del gobierno argentino. En 1997, una vez que la oposición al peronismo consiguió mayoría en el Senado, la poligonal fue descartada definitivamente, dándose lugar a un nuevo trazado. Este último demandó negociaciones secretas entre Guido di Tella y la oposición ante el riesgo de que emergieran exigencias de la opinión pública de no negociar con el gobierno chileno de hacerse públicas las negociaciones ya referidas.

En efecto, la propuesta del nuevo trazado demandó una acción intensiva de ambos Congresos para lograr los acuerdos. La diplomacia parlamentaria fue esencial para afianzar las negociaciones nacionales e internacionales (Lucero, 2007: 42-74). Esto permitió al Poder Ejecutivo de cada país asegurar rápidamente los acuerdos alcanzados a nivel legislativo (Lucero, 2007: 75-100). Luego, el 16 de diciembre de 1998 las partes firmaron el tratado que solucionó parcialmente la controversia al dejar pendiente la Sección B, que se encontraba en una indefinición cartográfica que se mantiene actualmente.

Por otra parte, el perfeccionamiento del ACE 16 estaba supeditado al avance de las negociaciones para solucionar la controversia de Campos de Hielo Sur. Al efecto se suscribieron 14 protocolos adicionales entre julio de 1995 y septiembre de 2002. De estos últimos, uno de los más críticos fue el de interconexión gasífera $-15^{\circ}$ protocolo adicional (7 de julio de 1995), que sustituye al $2^{\circ}$ protocolo (17 de julio de 1992)-, cuyo régimen pondrá a prueba la consistencia de la relación argentino-chilena en 2004. Además, es importante señalar que con estos acuerdos quedaron cubiertos todos los sectores de la economía de ambos países susceptibles de exportación de bienes, servicios e inversiones (BID-INTAL, 2002), si se considera el acuerdo sobre protección recíproca de inversiones (1995).

Los demás temas también estaban subordinados a la solución de Campos de Hielo Sur desde la perspectiva chilena. Por ejemplo, el Tratado de Integración y Complementación Minera recién entró en vigencia el 2000. Este acuerdo era esencial para Argentina, ya que se proyectaba que la minería se convertiría en el quinto sector más importante de su economía (Lucero, 2007: 90). Esta convención fue crucial también para la evolución del ACE 16, especialmente en el ámbito de la integración minera $-19^{\circ}$ (El Pachón) y $20^{\circ}$ (Pascua Lama) protocolos adicionales (29 de enero de 1997)-, la que generaba profundas expectativas en actores estatales y no estatales de ambos países de construir una asociación que permitiría el desarrollo de una minería conjunta que apuntalara los demás ámbitos de la relación bilateral (BID-INTAL, 2002: 115-33).

Una excepción importante al proceso de ralentización de la agenda bilateral fue el área de Seguridad y Defensa. En este sentido, mediante el memorándum de entendimiento (MOU) de 1995 se configuró el Comité Permanente de Seguridad Argentino-Chileno (COMPERSEG), las Reuniones 2+2 y las reuniones entre los Estados Mayores de la Defensa y Conjunto de ambos países. ${ }^{7}$ Estas entidades han contribuido a minimizar 
la percepción de amenaza de ambos países y desmantelar la desconfianza estructural -desde la imaginación geopolítica- de los actores políticos y estratégicos (Mendoza, 2004: 113-7), a partir de las MCM que le dieron forma (Fuentes y Álvarez, 2011: 528-9), y que comienzan con el Tratado de Paz y Amistad (Faundes, 2009: 57). Las visiones e ideas generadas en el proceso evolutivo de la agenda de Seguridad y Defensa se plasmaron en los libros de la defensa respectivos, dando cuenta de un proceso que muestra más bien una hipótesis de colaboración entre ambos países (Ministerio de la Defensa Nacional de Chile, 1997; Ministerio de Defensa de Argentina, 1999).

En el área de Integración Física, se desarrollaron proyectos de transporte internacional a partir del Plan Maestro General de Pasos Fronterizos (1992) y el Acta sobre Integración Física (1994), como los de los ferrocarriles Antofagasta-Salta, Zapala-Lonquimay y el Trasandino Central, además de otros que requirieron de mejoras a los CDF por medio del acta de 1997. También es factible mencionar que estos proyectos adquirieron un alto nivel de importancia para la integración del sistema de transportes del Cono Sur, tanto en materia de ferrocarriles como en carreteras y pasos fronterizos (Thomson, 1997: 39-50; Sant'Anna, 1997: 20 y 32-3). Dichas iniciativas, entre las otras que se generaron en las áreas ya señaladas, hicieron imprescindible un mayor acople entre el transporte marítimo y el terrestre (Sgut, 1997: 9-10) en el marco del MERCOSUR. Varios de estos proyectos fueron canalizados mediante el IIRSA (2000) tras la Cumbre de Jefes de Estado de Brasilia.

Así, la relación alcanzó su apogeo al avanzar notablemente la agenda bilateral ejecutada hasta entonces (Declaración Conjunta, 1998). Este proceso se afianzó tras la firma del Tratado de Campos de Hielo Sur y la promulgación en ambos países de todos los convenios y tratados pendientes de ratificación debido a la controversia. Así, se firmaron tratados y declaraciones presidenciales conjuntas que abarcaron el fomento a la seguridad militar (Declaración Presidencial Conjunta, 1999: 2) y la cooperación política y económica (2000) en el gobierno de Fernando de la Rúa (1999-2001). Además se emitieron tres declaraciones presidenciales conjuntas (2000) que reafirmaron los logros de la relación. No obstante, la política exterior del período de De la Rúa se caracterizó por mantener la continuidad de la agenda binacional, ya que era importante impulsar en esta fase la fórmula MERCOSUR ampliado, que buscó contener la presencia gravitatoria de Brasil en la región mediante la alianza con Chile (Simonoff, 2013: 26).

\section{CONSOLIDACIÓN Y CRISIS DE LA NUEVA AGENDA BILATERAL (2003-2007)}

La crisis económica argentina alcanzó su clímax en el período 2001-2003, lo que generó una profunda crisis política y social. Si bien aquella no interrumpe el desarrollo de la relación bilateral, sí mermó la evolución de la agenda durante el lapso 2001-2003, hasta la asunción de Néstor Kirchner como Presidente de Argentina (2003).

En el ámbito político, el instrumento que estuvo llamado a afianzar con mayor fuerza el desarrollo de la relación bilateral fue la Declaración Presidencial Conjunta de 2003, la que señala en sus puntos 1 y 3 : 
1.- Ambos Mandatarios renovaron su firme compromiso con la democracia, el estado de derecho, el pluralismo político, la participación plena de la ciudadanía en los sistemas políticos de los Estados, la solución pacífica de las controversias, el respeto a los derechos humanos y a las libertades fundamentales.

3.- Reafirmaron el compromiso con los objetivos de la Declaración del Milenio y con una renovación de las Naciones Unidas para que esta responda a los nuevos desafíos del mundo global a comienzos del siglo XXI.

Asimismo, destacaron la necesidad de seguir coordinando esfuerzos en el marco del Grupo de Trabajo de Composición Abierta, establecido por la Asamblea General para examinar todos los aspectos vinculados a la reforma del Consejo de Seguridad. Al respecto, señalaron que toda reforma debe adoptarse por un acuerdo general y teniendo en cuenta los equilibrios regionales, con el propósito de que el Consejo tenga una representación equitativa y un proceso de toma de decisiones más democrático que fortalezcan su rol de garante de la seguridad colectiva (Declaración Presidencial Conjunta de los Señores Presidentes de la República Argentina y de la República de Chile, 2003: 2).

Podemos inferir de la cita que existe, respecto de la agenda bilateral de Menem y Frei, una continuidad y coherencia discursiva en la relación argentino-chilena. Asimismo, se manifiestan diferencias sustantivas con el discurso en los dos períodos de Menem, siendo la más relevante el compromiso de desarrollar esfuerzos mancomunados en materia de política exterior, especialmente en los ámbitos de Política, Seguridad y Defensa ante la ONU, lo que implica mantener posiciones convergentes ante el Consejo de Seguridad en aquellas materias.

Además, en octubre de 2003 los ministros del Interior de ambos países acordaron el funcionamiento del régimen de Tránsito Vecinal Fronterizo desde el 1 de enero de 2004, y se observó una considerable evolución en el desarrollo de la agenda de integración física, al punto de que, ante el incremento de la actividad de los CDF y su relevancia, pasaron a agrupar a numerosos actores subnacionales y no estatales de nivel local y regional. Estos procesos permitieron constituir los Comités de Integración en 2006 (CDI) que desbordaron burocráticamente a los $\mathrm{CDF}$, al apuntalar estos numerosos proyectos de desarrollo económico locales y regionales de iniciativa privada, y estimular la discusión de asuntos comunes entre ambos países (Silva y Morán, 2010: 299-302).

Dentro del área económica, la relación se basó principalmente en el comercio gasífero. En efecto, la Crisis del Gas ocurre en un contexto en donde las necesidades energéticas de ambos países generaron condiciones para que colisionaran los intereses privados con el papel del Estado en el ámbito de la construcción de gasoductos y la distribución del gas, sin que los ajustes institucionales en ambos países ni los convenios alcanzados entre las partes permitieran la ejecución de los acuerdos y su cumplimiento a largo plazo (Gamboa y Huneeus, 2007: 83-116; Lorenzini, 2013: 44-5; Mares, 2006: 196).

Tras la crisis, la agenda bilateral comenzó a desarrollarse normalmente. La agenda generada desde el período Aylwin-Menem llegó a un nuevo nivel, ya que se constituyó 
la Fuerza de Paz Conjunta Combinada Cruz del Sur, lo que conllevó un alto nivel de cooperación militar, al definirse el organigrama, funciones, recursos y procesos de aquella (Memorándum de Entendimiento entre el Ministerio de Defensa de la República Argentina y el Ministerio de Defensa Nacional de Chile referente a la Fuerza de Paz Combinada "Cruz del Sur", 2006), para dejar a disposición de la ONU un contingente militar conjunto especializado en operaciones de paz que cuenta con un máximo de 1.400 efectivos (Scuticchio, 2015).

Lo anterior dio lugar a la suscripción de varios compromisos en los años siguientes (2006-2009). Se emitió la Declaración de Mendoza (2006) -Néstor Kirchner y Michelle Bachelet-, que calendarizó el desarrollo de la agenda bilateral, centrando los esfuerzos en los CDI, reuniones de ministros, entre otros. Además, se firmaron dos protocolos adicionales al Tratado sobre Integración y Complementación Minera (2006), una segunda Declaración Presidencial Conjunta (2006), y la Declaración Conjunta de Ministros (2006).

\section{LA AGENDA BILATERAL INSTITUCIONALIZADA (2008-2011)}

El ciclo desarrollado durante los mandatos de Michelle Bachelet y Cristina Fernández continuó profundizando los compromisos de la agenda bilateral. Por ejemplo, los aspectos más destacables de la Declaración Presidencial Conjunta de 2008 son dos: el primero alude a la reafirmación, por parte de ambas presidentas, de los valores que han sustentado la relación bilateral desde 1984, al destacar ambas

[...] con especial énfasis el escenario actual, treinta años después de aquel acontecimiento histórico [la firma del Tratado de Paz y Amistad de 1984], en el cual Argentina y Chile representan un modelo de integración estratégica, consolidada en valores comunes, como la paz, la democracia, la promoción y defensa de los derechos humanos y la justicia social, presupuestos necesarios para la construcción de una sociedad más equitativa en el marco de un desarrollo integral y sustentable (Declaración de Punta Arenas, 2008: 1).

Se puede deducir de la cita precedente que existe continuidad en los valores que inspiran el desarrollo de la agenda. No obstante, el segundo elemento novedoso dentro del discurso es que se pone énfasis en el valor estratégico que cada uno tiene para el otro. Si bien es cierto que el valor que cada uno tiene para el otro se dejó ver en la ejecución de la agenda, no existían declaraciones que manifestaran abiertamente el carácter estratégico de la relación con el vecino. Asimismo, se deja ver una agenda bilateral con más énfasis en su multidimensionalidad que el mostrado durante los dos períodos de Menem (19891999), y que exhibe un nivel mayor de compromiso respecto de profundizar el estatus de la relación bilateral, al denominarla como un modelo de integración estratégica.

Por otra parte, en agosto del 2009 se ejecutó la II Reunión Binacional de Ministros (2009), que abordó lo obrado en la agenda bilateral en todas las materias desde 1990. Además, continuaron los trabajos para constituir la Fuerza de Paz Conjunta y Combinada Cruz del Sur, la que fue presentada durante el acto de firma de la Declaración de Punta Arenas (2008) en los siguientes términos: 
Su ámbito de acción constituye un notable avance cualitativo en materia de defensa, y evidencia los niveles de entendimiento que han permitido pasar de una etapa de medidas de confianza mutua a una de integración y cooperación, convirtiendo a Cruz del Sur en un valioso aporte conjunto a la paz y seguridad internacionales, lo que ha sido posible a través de la labor desarrollada desde 1995 por los Ministerios de Defensa y de Relaciones Exteriores (Declaración de Punta Arenas, 2008: 2).

Este último punto es relevante, por cuanto confirma lo comprometido por ambos países en la Declaración Presidencial Conjunta de 2003, con relación a mantener aspectos centrales de la política de seguridad y defensa de Argentina y Chile bajo un régimen de alta coordinación en materia de operaciones de paz, precedida por ciertas directrices comunes de política exterior acerca del tema en comento. Esto supone una profundización respecto de la primera acción conjunta de contingentes argentinos y chilenos en el marco de la UNFICYP (2006) (Várnagy, 2010: 189).

En el ámbito de Economía, una vez concluidos los episodios de tensión y desconfianza producidos por la Crisis del Gas, ambos países se focalizaron en ampliar el Tratado de Integración y Complementación Minera mediante la firma de los protocolos adicionales de los proyectos La Flecha y Vicuña -provincia de San Juan y región de Atacama-. Por otra parte, se formó la Comisión Binacional de Comercio, Inversiones y Relaciones Económicas en 2008, momento a partir del cual emerge una institución binacional destinada a dirigir un proceso de integración económica en la formación de cadenas de valor en la producción de bienes y servicios entre ambos países (Lorenzini, 2013: 47-8).

En cuanto al área de Integración Física, se firmaron dos protocolos adicionales, los que abarcan el Túnel de Baja Altura Agua Negra y la constitución de una entidad binacional para el proyecto de Túnel de Baja Altura-Ferrocarril Trasandino Central durante los años 2010 y 2011. Esto último convierte a aquellos proyectos en los puntales del proceso de integración física si consideramos, por ejemplo, que el Ferrocarril Trasandino podría transportar, de acuerdo con el proyecto del Corredor Bioceánico Aconcagua, flujos de 15 millones de toneladas de carga anuales (etapa 1), para luego aumentar a 20 millones de toneladas (etapa 2), hasta alcanzar en el largo plazo una capacidad de 70 millones de toneladas (etapa 3) (Lacoste y Jiménez, 2013: 86-89).

\section{EL TRATADO DE MAIPÚ: ACTUALIZACIONES, CONSTANTES Y CAMBIOS}

En su Declaración de Principios, el Tratado de Maipú señala:

CONSIDERANDO los lazos de hermandad entre Chile y la Argentina, reforzados por las gestas históricas comunes, el legado de valores compartidos, las manifestaciones de una cultura propia, la vecindad geográfica y la afinidad entre sus habitantes, todo lo cual sienta las bases destinadas a profundizar y consolidar las relaciones bilaterales existentes y su proyección hacia un futuro promisorio para las Partes (Tratado de Maipú, 2009: 2).

De acuerdo con la cita, los gobernantes de ambos países declaran la existencia de un destino manifiesto conjunto, contexto en el que una alianza argentino-chilena cada vez más 
profunda es el destino que ambos países comparten, lo que representa la consolidación de la relación en el plano discursivo bajo un modelo de integración estratégica.

Por otra parte, cabe señalar que el Tratado de Maipú creó solamente los Encuentros Presidenciales, y el Sistema de Consultas Permanentes de Ministros de Relaciones Exteriores. Asimismo, el Consejo Empresarial Binacional Permanente (2005) -formalizado solo en el Tratado de Maipú- recoge las necesidades del sector privado dentro de las iniciativas y los acuerdos económicos que ambos Estados alcancen. Así, el Tratado insta a ambos países a negociar permanentemente, de modo tal que cuando las instituciones actúen, lo hagan para rubricar los acuerdos generados anualmente, revisar la concordancia de los resultados de corto plazo de la relación con aquellos de largo plazo, corregir las falencias del modelo de integración y coordinar las políticas exteriores de ambos países dentro de las cuatro áreas clave de política ya examinadas.

Un tercer factor consiste en que el Tratado, más que innovar en lo institucional, establece un sistema de coordinación multinivel entre las instituciones binacionales que ya existían con anterioridad a aquel, lo que permite incluir los resultados de los acuerdos alcanzados por los CDI en las negociaciones del Comité Binacional de Cooperación Económica e Integración Física y la Comisión Binacional de Comercio, Inversiones y Relaciones Económicas (art. 18). Igualmente, los CDI pueden entregar sus propuestas a la Comisión Parlamentaria Conjunta y a los Ministros de Relaciones Exteriores (arts. 15 al 19), lo que muestra un proceso de conexión de alta relevancia entre política subnacional (Silva y Morán, 2010: 259-303) y la política exterior nacional de Argentina y Chile.

En relación con la conexión entre los cuatro ámbitos de política señalados al comienzo de este trabajo, es posible señalar que, además de la red descrita en el párrafo anterior, también se estableció un sistema de coordinación, asesoría y apoyo entre la Comisión Parlamentaria Conjunta y los Encuentros Presidenciales, el Comité Binacional de Cooperación Económica e Integración Física y el Comité Binacional de Comercio, Inversiones y Relaciones Económicas (arts. 13 y 14).

Por otra parte, mediante sus protocolos complementarios, el Tratado de Maipú define como proyectos de interés público para ambos países la construcción de los túneles de baja altura del Ferrocarril Trasandino Central y del Paso Agua Negra, lo que convierte a estos dos proyectos, junto con los CDI y el Comité Binacional de Cooperación Económica e Integración Física, en los principales ejes del proceso de integración física binacional.

Finalmente, en lo institucional, el Tratado de Maipú recoge la práctica de ambos países en la conducción de sus relaciones, consolidando un sistema institucional binacional autónomo asentado en cuatro pilares (Tablas 1 y 2): 1) la concentración de casi todas las funciones de las instituciones binacionales en el Poder Ejecutivo de ambos países; 2) la coordinación multinivel de las instituciones binacionales y los actores no estatales en las áreas de Política, Integración Física y Economía; 3) el continuo desarrollo y perfeccionamiento de las instituciones clave en el área de Seguridad y Defensa con un grado mayor de coherencia y continuidad respecto de las demás áreas clave de política, y; 4) la incorporación de veto players en cada una de dichas áreas, lo que permite, en principio, que cada área se desarrolle en un marco de estabilidad y cambios graduales. 


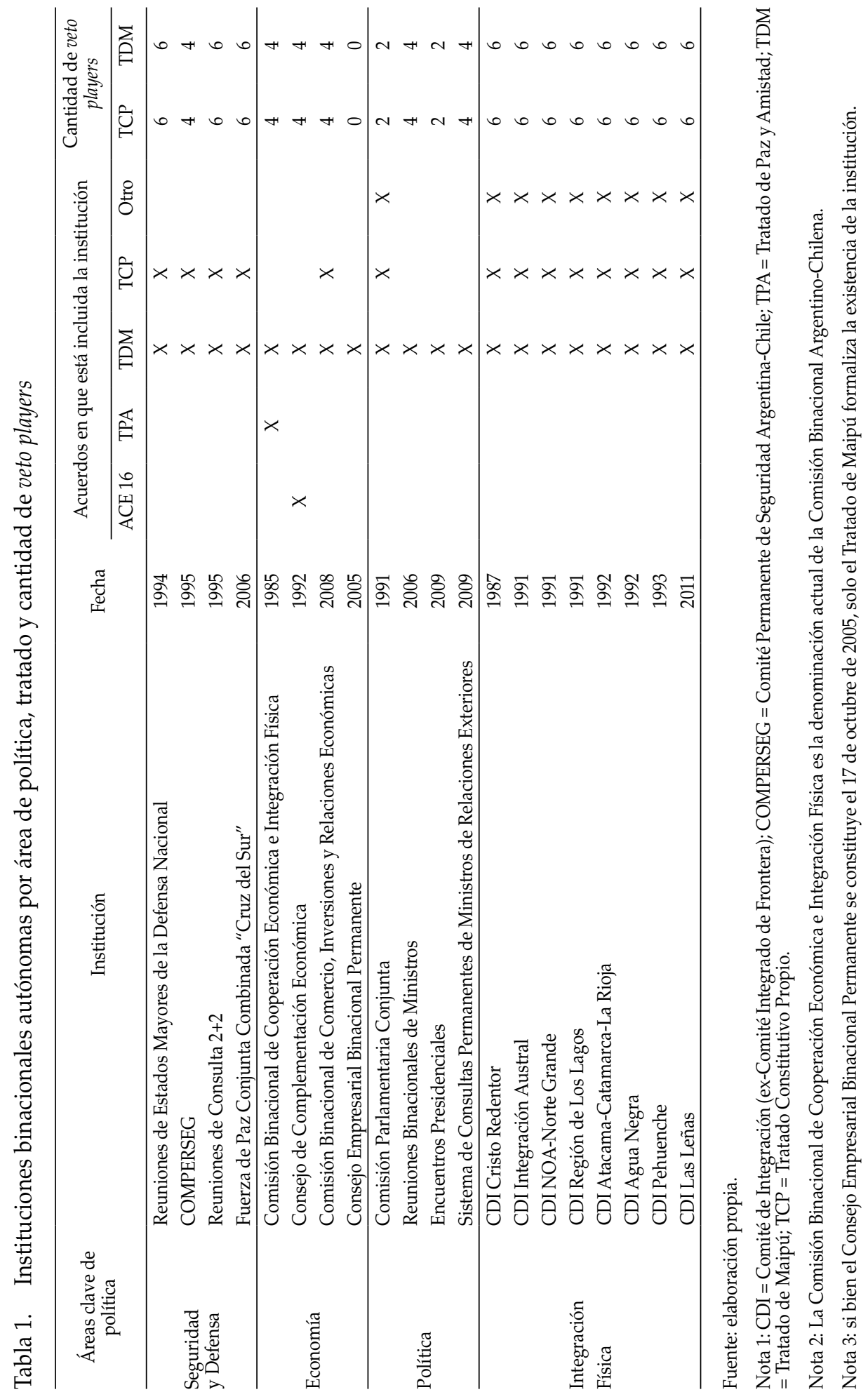


DESDE EL TRATADO DE PAZ Y AMISTAD DE 1984 AL TRATADO DE MAIPÚ DE 2009: UN PROCESO EVOLUTIVO INSTITUCIONALMENTE...

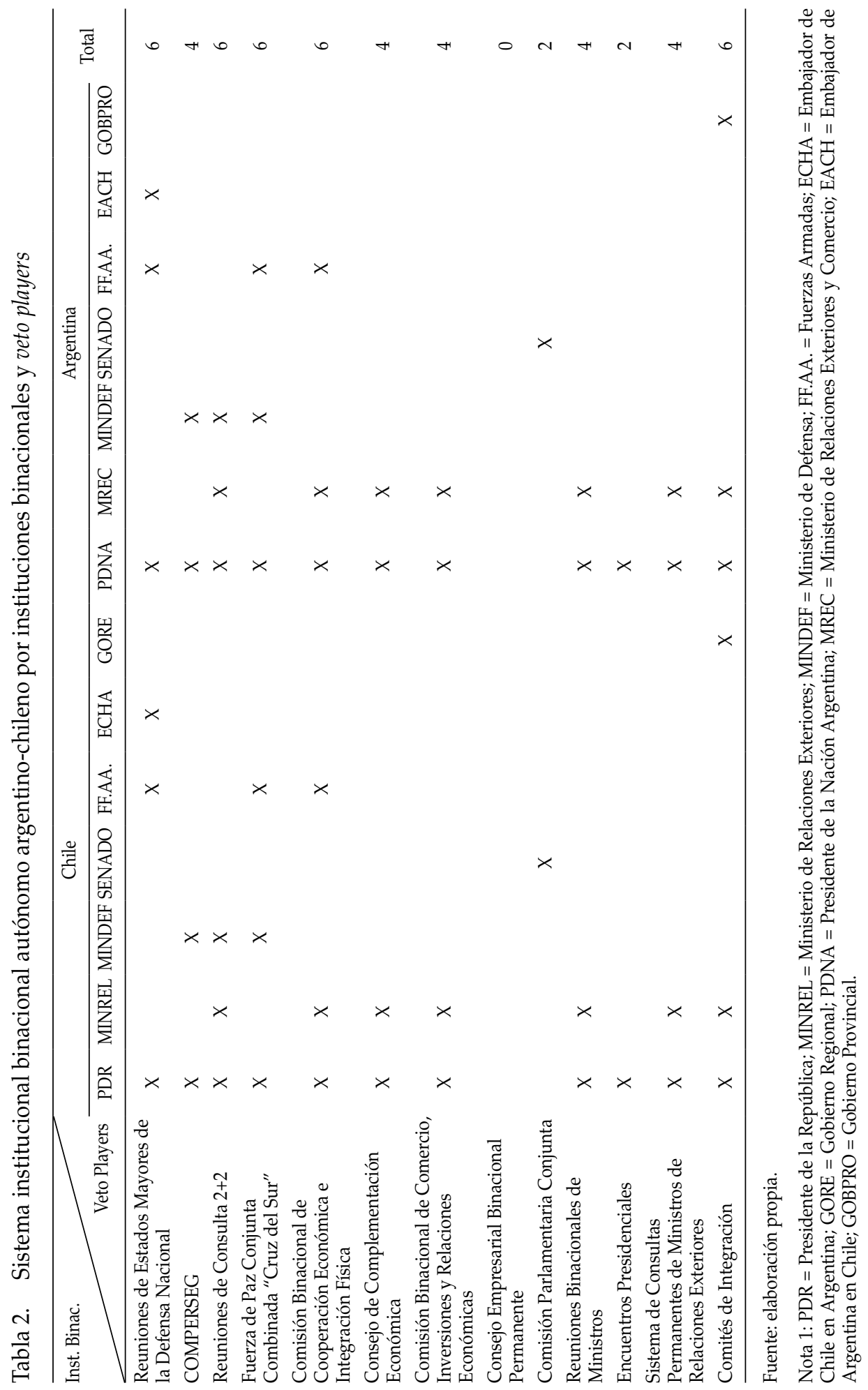




\section{CONCLUSIONES}

Dentro de las cuatro etapas de la relación argentino-chilena se observa un proceso de evolución del discurso geopolítico sobre la base de la desintegración de los factores que producen la desconfianza entre ambos países y la materialización de instituciones binacionales. Este discurso, al exigir interacciones y revisiones constantes, ha hecho que cada institución binacional contribuyera a incrementar la confianza mutua, lo que ha sido la tónica de todo el período. Empero, para comprender mejor el desarrollo de la relación, revisaremos cada una de las cuatro etapas contempladas en este artículo.

En primer lugar, la etapa de la agenda histórica (1989-1994) se caracterizó porque las cuatro áreas clave de política se desarrollaron sin mayores trastornos, tras la separación de Laguna del Desierto de los demás temas de la agenda bilateral y la postergación de Campos de Hielo Sur para cuando el litigio quedase jurídicamente resuelto.

La segunda etapa (1995-2002) estuvo marcada por el desarrollo de la controversia de Campos de Hielo Sur. A diferencia de la primera etapa, en esta el difirendo sí condicionó el desarrollo de la agenda en tres de las cuatro áreas claves de política, ya que no existía un tercero que le diera solución. En efecto, podemos concluir que la negociación directa implica riesgos como el estancamiento de la agenda en su conjunto si no se alcanza una solución al problema central, pero también permite generar soluciones transaccionales que acaban por fomentar la confianza entre los actores de las élites de ambos países, que fue lo ocurrido en este caso entre 1998 y 2001.

En la tercera etapa (2003-2007) ocurrió algo similar con la Crisis del Gas, con la diferencia de que esta tuvo una mayor gravedad en la relación, en razón de los niveles de confianza alcanzados hasta esa fecha y la voluntad geopolítica comprometida por ambos países para hacer posible la integración gasífera.

En la cuarta etapa (2008-2011) se observa nuevamente un desarrollo sin mayores sobresaltos de las cuatro áreas clave, después de la "desgasificación" de la relación. Asimismo, se alcanza un punto muy prolífico en la evolución del discurso geopolítico al declararse un destino manifiesto de ambos países y la necesidad de cada uno para el desarrollo del otro bajo la lógica del modelo de integración estratégica, lo que no se observó en ninguno de los discursos previos a la firma del Tratado de Maipú. Así, la imagen del otro mejoró sostenidamente en todo el período.

Finalmente, el Tratado de Maipú representa un punto cúlmine en la relación argentinochilena al producir este último tres efectos en dicha relación: 1) recogió la práctica y la experiencia de ambos países en su relación con el otro mediante la actualización de los principios de la relación y sus instituciones; 2) generó un sistema de pesos y contrapesos entre las instituciones binacionales autónomas recogidas en el tratado, y;3) la definición de una cantidad creciente de veto players en cada una de las cuatro áreas clave de política, lo que permite, teóricamente, dotar a este sistema institucional binacional autónomo de una lógica de estabilidad y gradualidad de los cambios que funcione con una considerable independencia de los problemas de política interna de Argentina y de Chile. Son estos elementos los que permiten definir al Tratado de Maipú como el punto final de la transición que se inició con el Tratado de Paz y Amistad. 


\section{REFERENCIAS}

\section{Fuentes primarias}

Biblioteca Digital de Tratados del Ministerio de Relaciones Exteriores y Comercio de Argentina. 1990. "Declaración Conjunta argentino-chilena sobre la Relación Bilateral". Santiago de Chile. 29 de agosto. 1991. "Acuerdo entre el Gobierno de la República Argentina y el Gobierno de la República de Chile para precisar el límite en la zona comprendida entre el monte Fitz Roy y el cerro Daudet". Buenos Aires. 2 de agosto. 1991. “Declaración Presidencial Conjunta sobre Límites entre la República Argentina y la República de Chile". Buenos Aires. 2 de agosto. 1994. "Acuerdo por canje de notas argentino-chileno estableciendo un mecanismo de consulta". Santiago de Chile. 26 de agosto. 1998. "Declaración Conjunta". Buenos Aires. 15 de diciembre. 1999. "Declaración Presidencial Conjunta sobre fomento de la confianza y la seguridad". Ushuaia. 16 de febrero. 1999. "Declaración Presidencial Conjunta entre la República Argentina y la República de Chile. Santiago de Chile". 20 de agosto. 2003. "Declaración Presidencial de El Calafate". El Calafate. 29 de agosto.

. 2006. "Memorándum de Entendimiento entre el Ministerio de Defensa de la República Argentina y el Ministerio de Defensa Nacional de la República de Chile referente a la Fuerza de Paz Combinada "Cruz del Sur'”. Buenos Aires. 4 de diciembre.

2008. Declaración de Punta Arenas. Punta Arenas. 4 de diciembre.

Ministerio de Defensa de Argentina. 1997. "Libro de la Defensa Nacional de Chile”. Chile, Santiago de Chile: Ministerio de Defensa de Argentina. 1999. "Libro Blanco de la República Argentina”. Argentina, Buenos Aires. Tratado de Maipú. 2009. Santiago de Chile. 29 de octubre.

Tribunal Arbitral Internacional. 1995. "Sentencia del 13 de octubre de 1995. Solicitud de revisión y de interpretación en subsidio planteada por Chile respecto de la Sentencia del 21 de octubre de 1994". Río de Janeiro.

\section{Fuentes secundarias}

Agnew, John. 2005. Geopolítica: una re-visión de la política mundial. Madrid: Trama Editorial.

Banco Interamericano del Desarrollo (BID)-Instituto para la Integración de América Latina y el Caribe (INTAL). 2002. Perspectivas y Desafíos del Proceso de Integración Argentino-Chileno a Diez Años del $A C E$ 16. Buenos Aires: BID-Consejo Argentino para las Relaciones Internacionales (CARI)-Centro Latinoamericano para las Relaciones con Europa.

Ceresole, Norberto. 1987. "Geohistopolítica sudamericana". En Argentina: sobre transiciones y decadencias. Cinco ensayos geopoliticos para la re-interpretación de la realidad argentina, editado por Norberto Ceresole. Buenos Aires: ILCTRI, 85-119.

Cicerchia, Ricardo y Eduardo Cavieres (coords). 2012. Argentina-Chile, Chile-Argentina: 1820-2010. Desarrollos Políticos, Económicos y Culturales. Valparaíso: Ediciones Universitarias de Valparaíso.

Colacrai, Miryam. 2010. "El tejido de relaciones bilaterales desde el ámbito subnacional. Desarrollo de una innovadora interdependencia entre la Argentina y Chile". En La Politica Internacional Subnacional en América Latina, editado por Luis Maira. Buenos Aires: Libros del Zorzal, 305-327.

Eissa, Sergio. 2010. Hielos Continentales. La política exterior argentina en los 90, Buenos Aires: Centro Argentino de Estudios Internacionales. E-Book $\mathrm{N}^{\circ} 45$.

Escudé, Carlos. 1992. Realismo periférico: fundamentos para la nueva política exterior argentina. Buenos Aires: Editorial Planeta.

Faundes, Cristián. 2009. "La fuerza combinada Cruz del Sur, un análisis desde las medidas de confianza mutua". Estudios Avanzados 12: 53-74.

Fuentes, Claudio y Gonzalo Álvarez. 2011. "Argentina e Chile: mudança de paradigma?". Contexto Internacional 33 (2): 521-540. 
Gallois, Pierre. 1992, Geopolítica. Los caminos del poder. Madrid: A.G Grupo S.A.

Gamboa, Ricardo y Carlos Huneeus. 2007. "La interconexión gasífera Chile-Argentina: objetivos y actores".

Estudios Internacionales 157: 83-116.

Gómez, Héctor. 1977. Teoría y doctrina de la geopolítica. Buenos Aires: Editorial Astrea.

Illanes, Javier. 2003. El arbitraje de Laguna del Desierto. Santiago de Chile: RIL Editores.

Jiménez, Diego. 2012. La aspiración geopolítica argentina en la relación argentino-chilena: una mirada desde Chile (1990-2010). Tesis de pregrado inédita. Concepción: Universidad de Concepción.

Kastner, Scott. L. y Chad Rector. 2003. "International Regimes, Domestic Veto Players, and Capitals Control Policy Stability". International Studies Quarterly 47 (1): 1-22.

Lacoste, Pablo. 2000. "El concepto de zonas de influencia y su aplicación en las relaciones entre Argentina y Chile". Estudios Internacionales 131-132: 65-92.

2003. La imagen del otro en las relaciones de la Argentina y Chile (1534-2000). Santiago de Chile: Universidad de Santiago de Chile-Fondo de Cultura Económica.

Lacoste, Pablo y Diego Jiménez. 2013. “Transporte internacional y actores subnacionales: la provincia de Mendoza y la resurrección del ferrocarril trasandino entre Argentina y Chile". Si Somos Americanos XIII (1): 65-92.

Lorenzini, María Elena. 2013. “Las relaciones argentino-chilenas 2008-2011, ¿realidad o ficción de la 'alianza estratégica"?". Si Somos Americanos 13 (1): 39-64.

Lucero, Mariel. 2007. "El poder legislativo en la definición de la política exterior argentina. El caso de los Hielos Continentales Patagónicos". Cuadernos Política Exterior Argentina 90: 1-124.

Maira, Luis (comp.). 2010. La Política Internacional Subnacional en América Latina. Buenos Aires: Libros del Zorzal.

Mansfield, Edward D.; Helen V. Milner y Jon C. Pevehouse. 2007. "Vetoing Co-operation: The Impact of Veto Players on Preferential Trade Agreements". Journal of Political Science 37 (3): 403-432.

Manzano, Karen. 2015. "Representaciones geopolíticas: Chile y Argentina en Campos de Hielo Sur". Estudios fronterizos 17 (33): 83-114.

Mares, David. 2006. "Natural gas pipelines in the Southern Cone". In Natural Gas and Geopolitics. From 1970 to 2040, editado por Victor, David. G., Amy M. Jaffe and Mark H. Haye. Cambridge: Cambridge University Press, 169-201.

Marini, José Felipe. 1985. El conocimiento geopolítico. Buenos Aires: Editorial Círculo Militar.

Mendoza, Juan Eduardo. 2004. “El Tratado de Paz y Amistad de 1984, la cooperación militar y los problemas limítrofes entre Chile y Argentina". Universum 19 (1): 110-121.

Muñoz, Heraldo (ed.). 1989. Chile: política exterior para la democracia. Santiago de Chile: Pehuén Editores. Palomares, Gustavo. 2006. Relaciones Internacionales en el siglo XXI. Madrid: Editorial Tecnos.

Pinto, Julio. 2010. “La provocadora lectura de George L. Mosse acerca del sentido de los nacionalismos. Su importancia teórica para la comprensión de los populismos latinoamericanos". Revista SAAP 4 (2): $145-160$.

Rhodes, Edward. 1994. “Do Bureaucratic Politics Matter? Some Disconfirming Findings from the Case of the U.S Navy". World Politics 47 (1): 1-41.

Rodríguez, Eduardo. 2006. Chile, país puente. Santiago de Chile: RIL Editores.

Runza, Ricardo. 2004. Análisis y evaluación de las relaciones argentino-chilenas de defensa, desde la perspectiva de la Comisión Permanente de Seguridad (COMPERSEG). Hamburgo: Universidad de Hamburgo, 1-20. Informe de investigación.

Russell, Roberto. 1992. La política exterior argentina en el nuevo orden mundial. Buenos Aires: Grupo Editor Latinoamericano.

2010. "El Estado y los actores gubernamentales no centrales: una relación complementaria". En La Politica Internacional Subnacional en América Latina, editado por Luis Maira. Buenos Aires: Libros del Zorzal, 83-106.

Russet, Bruce and Harvey Starr. 1990. "Individuals and Their Role in Creating Policy". En Theories of International Relations, editado por James. A. Hursch. Washington D.C.: National Defense University, 53-73. 
Sant'Anna, José. 1997. Transporte terrestre. Serie Integración en el Transporte en el Cono Sur. Buenos Aires: BID-INTAL.

Scuticchio, Pablo. 2015. “Una misión de paz sudamericana para Colombia?”. Foreign Affairs Latinoamérica (23 de abril). En: http:/ / revistafal.com/una-mision-de-paz-sudamericana-para-colombia/

Sgut, Martín. 1997. Puertos y vías navegables. Serie Integración en el Transporte en el Cono Sur. Buenos Aires: BID-INTAL.

Silva, Verónica y Carlos Morán. 2010. "Relaciones Subnacionales. El Caso de Chile y Argentina”. En La Política Internacional Subnacional en América Latina, editado por Luis Maira. Buenos Aires: Libros del Zorzal, 259-303.

Simonoff, Alejandro. 2013. “Una visión estructural de la política exterior argentina y el rol de Chile desde el proceso de democratización de 1983". Si Somos Americanos 13 (1): 15-38.

Thomson, Ian. 1997. Los ferrocarriles y su contribución al comercio internacional. Serie Integración en el Transporte en el Cono Sur. Buenos Aires: BID-INTAL.

Tsebelis, George. 1995. “Decision Making in Political Systems: Veto Players in Presidentialism, Parliamentarism, Bicameralism and Multipartyism". British Journal of Political Science 25 (3): 289-325.

Uc, Pablo. 2008. “El discurso geopolítico del petróleo como representación espacial de la economía política internacional". Nueva época 58: 109-133.

Valenzuela, Mario. 1999. El enigma de la Laguna del Desierto. Una memoria diplomática. Santiago de Chile: LOM Ediciones.

Van Klaveren, Alberto. 1988. "El Análisis de la Política Exterior Latinoamericana: Perspectivas Teóricas". En Análisis y Formulación de la Política Exterior, compilado por Programa de las Naciones Unidas para el Desarrollo (PNUD)-Consejo Económico para América Latina y el Caribe (CEPAL). Santiago de Chile: PNUD-CEPAL, 25-60. Documento de Trabajo No 1.

Várnagy, Tomás. 2010. “Naciones Unidas y regionalismo: 'Cruz del Sur', una fuerza de paz combinada argentino-chilena". Cuadernos de Marte 1 (0): 177-208.

Diego Jiménez Cabrera es magíster en Estudios Internacionales de la Universidad de Santiago de Chile y doctorando en Estudios Americanos especialidad en Estudios Internacionales de aquella universidad. Asimismo, es miembro de la Asociación Chilena de Especialistas Internacionales (ACHEI). E-mail: diego.jimenez.cabrera@gmail.com. 
